\title{
P80 SRM LOW TORQUE FLEX-SEAL DEVELOPMENT - THERMAL AND CHEMICAL MODELING OF MOLDING PROCESS
}

\author{
C. Descamps, E. Gautronneau, G. Rousseau, \\ and M. Daurat
}

\begin{abstract}
The development of the flex-seal component of the P80 nozzle gave the opportunity to set up new design and manufacturing process methods. Due to the short development lead time required by VEGA program, the usual manufacturing iterative tests work flow, which is usually time consuming, had to be enhanced in order to use a more predictive approach. A newly refined rubber vulcanization description was built up and identified on laboratory samples. This chemical model was implemented in a thermal analysis code. The complete model successfully supports the manufacturing processes. These activities were conducted with the support of ESA/CNES Research \& Technologies and DGA (General Delegation for Armament).
\end{abstract}

\section{INTRODUCTION}

For many years, the development and qualification of new flex-seals for several generations of solid rocket motors (SRM) have been relying on an experimental approach based on numerous tests and full-scale manufacturing components. As an important part of the new European Small Launcher VEGA program, the development of the P80 FW SRM aims to:

- the maturation of "low-cost" technologies for the next generation of large SRM; and

- the qualification of the first stage of the VEGA small launcher.

In 2001, Snecma Propulsion Solide (SAFRAN Group) responsible for the P80 nozzle, started the development of the flex-seal component. This component is considered as a critical part of the nozzle and its development gives the opportunity to set up new design and manufacturing process methods.

Based on a new design, the P80 flex-seal features new materials in order to reduce cost as well as actuation power, namely:

This is an Open Access article distributed under the terms of the Creative Commons Attribution-Noncommercial License 3.0, which permits unrestricted use, distribution, and reproduction in any noncommercial medium, provided the original work is properly cited. 
- thermal self-protection due to shims overlengths;

- low shear-modulus rubber;

- glass-epoxy shims; and

- new environmentally friendly bonding agents.

Additionally, due to the short development lead time required by VEGA program, the usual manufacturing iterative tests work flow which is usually timeconsuming, had to be enhanced in order to use a more predictive approach. Due to the availability of powerful thermal computer code, coupled with a newly refined rubber vulcanization model, a complete two-dimensional (2D) model was built up to help solving manufacturing difficulties. This model includes:

- the flex-seal simulation with a detailed mesh describing rubber pads, composite material shims, and metallic end-fittings,

- the molding tooling, taking into account its heat resistors and its temperature sensors.

A short implementation using subscale hardware and the corresponding model had been previously developed before the availability of the design and 2D model at P80 SRM scale. This preliminary activity allowed one to identify the relevant design modeling, parameters, and values to address.

The next step consisted in upgrading and tuning the model to the P80 design and manufacturing environment. This simulation code was very helpful in defining:

- the temperature range and limits inside the molding tooling with respect to the material requirements (rubber and bonding agent); and

- the power distribution requirements as well as the temperature monitoring and control management all along the vulcanization cycle.

Relying on this predictive approach, laboratory tests have started early in the development schedule, with the advantage of reducing the most important risks at a preliminary step where no costly hardware was committed on.

\section{FLEX-SEAL DESCRIPTION}

The thrust vector control is obtained by steering the nozzle using actuator-driven ball and socket system also called flexible bearing such as in Ariane 5 SRM. The flex-seal may be depicted as a stack of spherical-shaped shims and rubber pads (Fig. 1). The rubber sheets are chemically bonded to the rigid inserts, using adhesive agents laid on the inserts, which react during the molding and vulcanization processes. The use of glass-epoxy shims, instead of metallic ones, leads to a self-insulated flexible bearing. 


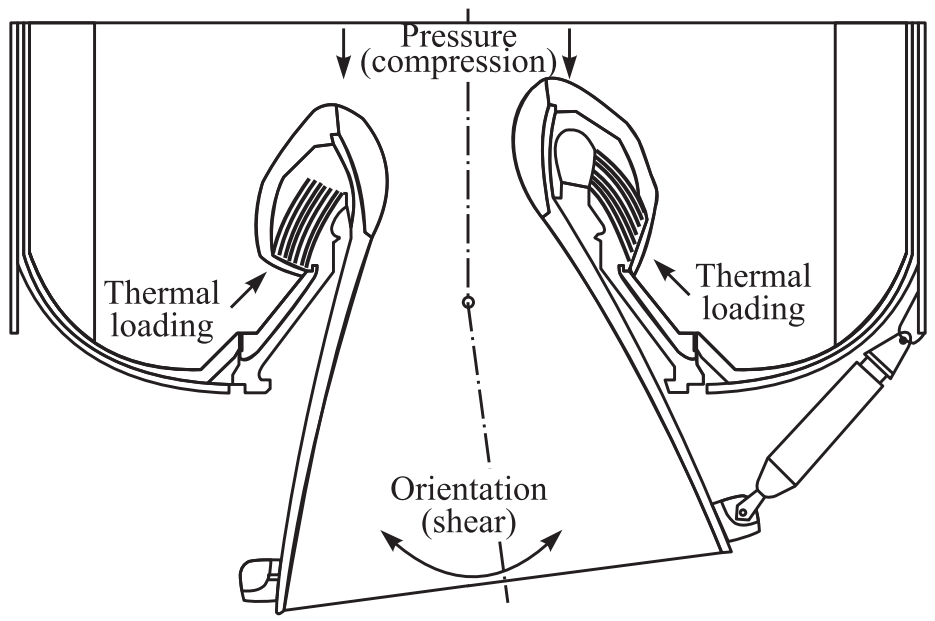

Figure 1 Flex-seal bearing principle

\section{MANUFACTURING PROCESS AND SUBSCALE PRELIMINARY MODELING}

\subsection{Manufacturing Process}

The stack of shims and rubber pads is obtained by means of a compression or transfer molding process. In both cases, the shims are maintained in the heating mold by combs. These combs determine the final thickness of the rubber pads. Bonding agents are applied on both faces of the shims, and then a layer of elastomer is applied above by means of an air-slip process. The mold is equipped with several resistive heating zones. The heating cycles usually involve several stages. The first ones aim to heat the remaining elastomer and to decrease its viscosity so that it may be easily transferred from the transfer pot to the injection channels. The second ones are effective curing stages.

\subsection{Subscale Preliminary Thermal Modeling}

The thermal modeling capabilities were tested against data available on a smallscale mold for flex-seal bearing. This development mold is somewhat similar to those used for full-scale flex-seal manufacturing (Fig. 2). For the model, the geometry is supposed to follow an axial symmetry, even if in reality, the axisymetry is broken by the existence of injection channels. These injectors were neglected because their volumes do not justify a significant alteration of the thermal behavior of the mold. The heating system of this mold consists in six independent 


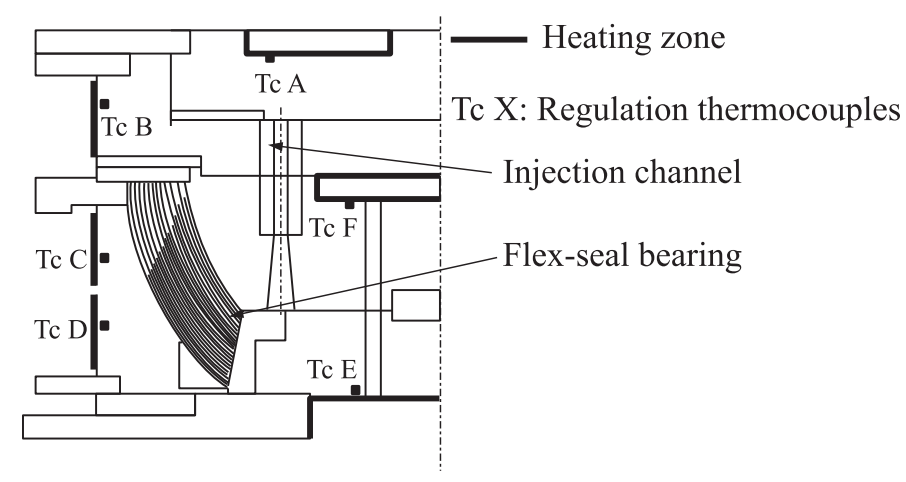

Figure 2 Representative scheme of flex-seal bearing development mold

resistive heating zones with different historical set points. The different parts of the mold consist in materials for which thermal properties $\left(\rho, C_{p}\right.$, and $\lambda$ as functions of temperature) are usually well known, except for the elastomeric pads. In order to estimate the dependence of heat capacity and conductivity on temperature, elastomer bricks were shaped and equipped with several thermocouples. The bricks were cured in an autoclave with nonisothermal cycle. The exact positions of the thermocouples were determined by an X-ray mean. The recorded thermal histories were used with an inverse identification method to estimate the unknown thermophysical properties of the elastomer. For this purpose, a Levenberg-Marquardt [1] method was applied to a thermal-dependent one-dimensional heat transfer equation. The protocol may be performed on both cured and uncured elastomer because they exhibit different thermal dependences. Specifically, uncured elastomer exhibits a small exothermic effect that may be associated with vulcanization effects. The errors between experimental data and those predicted by the model are usually smaller than one degree.

The thermal analysis that was carried out takes into account the main features of the compression molding process. In all the simulations, the mold was assumed to be in a closed position.

The preliminary modeling tests made it possible to conclude that the heating system on this mold could not be considered as perfect. So, historical set points are not representative of the real temperature of the resistive heating systems. One of the main reasons that may be invoced is the small scale of the mold. This prevents each heating zone from being independent when different temperature levels are set. In this case, conventional Dirichlet conditions for the model are not adequate. In order to correctly model the thermal behavior of the mold, the regulation was simulated in a simple but effective way: for each heating zone, the temperature calculated at the previous time step at located regulation point was compared to the corresponding thermal set-point. The nominal power was used 
as a source term for the entire heating zone in energy conservation equation if the calculated temperature was lower than the temperature to be reached; otherwise, no source term was used.

Heat losses were also taken into account by the mean of a natural convection coefficient, which was evaluated with the McAdams correlation for the Nusselt number [2]. The press itself was meshed, so that thermal losses through insulators were treated.

First, thermal simulations

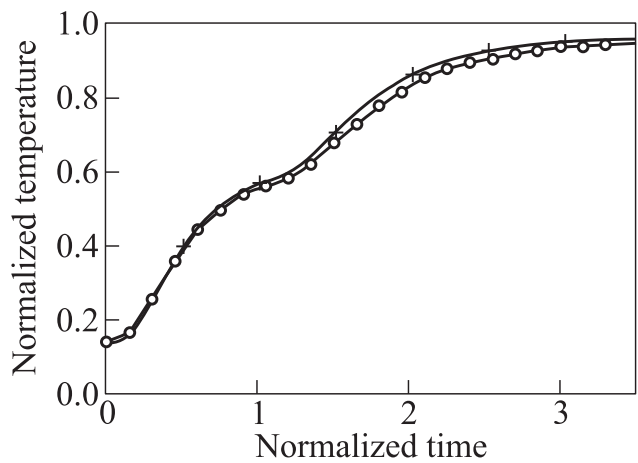

Figure 3 Experimental and simulated thermal histories on shim thermocouple in the development mold were performed for a configuration of a classical flex-seal bearing with an already developed elastomer and steel shims. Numerous case studies were treated for which different hypotheses on air gap localization between tooling equipments were tested to determine the most representative configuration. Calculated thermal histories in the mold and in the rubber pads were compared to the experimental ones. For the thermocouples located in the mold, there was a very good agreement. In the rubber pads where position of the thermocouple was not so precise, the agreement was nevertheless good.

In a second step, simulations were done with the same mold but with the newly developed low-torque flex-seal bearing with glass-epoxy shims and rubber pads and different curing cycles. The good agreement between calculated and experimental thermal histories made it possible to consider the model as valid (Fig. 3).

\section{CHEMICAL MODELING OF RUBBER-CURING}

\subsection{Sulphur Vulcanisation of Rubber}

The rubbers used for their mechanical properties in flexible bearings are usually cross-linked with a sulphur-based vulcanization agent. Vulcanization confers the required elastomeric properties to the rubber through the mean of monoor polysulfidic cross-links. The classical systems for sulphur vulcanization of natural rubber not only involve sulphur itself, but at least an activator and an accelerator. The accelerator may be chosen in the sulfenamide classes, which are also an important source for sulphur. Chemical reactions occurring during 


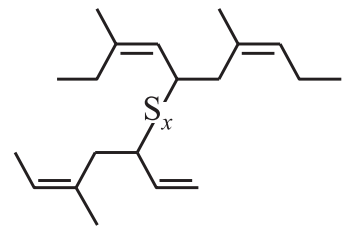

(a)

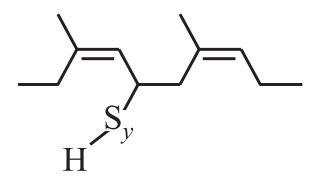

(c)

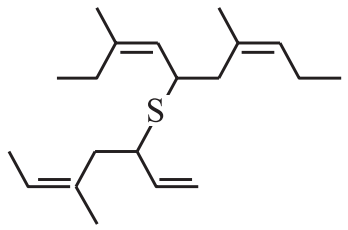

(b)

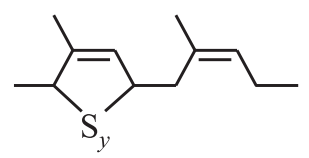

(d)

Figure 4 Typical sulfidic structures obtained during vulcanization process

curing lead to a complex three-dimensional (3D) structure for the rubber, and detailed mechanisms are not yet clearly identified. Some probable structures are depicted in Fig. 4.

Several techniques may be used in order to follow the structural evolution of the material. One of the most convenient consists in rheometric measurements. Under the hypothesis of small deformations, shear modulus is proportional to the concentration of sulfidic links between different polymer chains. Typical rheometric curves for sulphur vulcanization look like Fig. 5. The first stage corresponds to the prelinking chemistry or accelerator chemistry. The increase in shear modulus is related to the effective formation of sulfidic cross-links. The

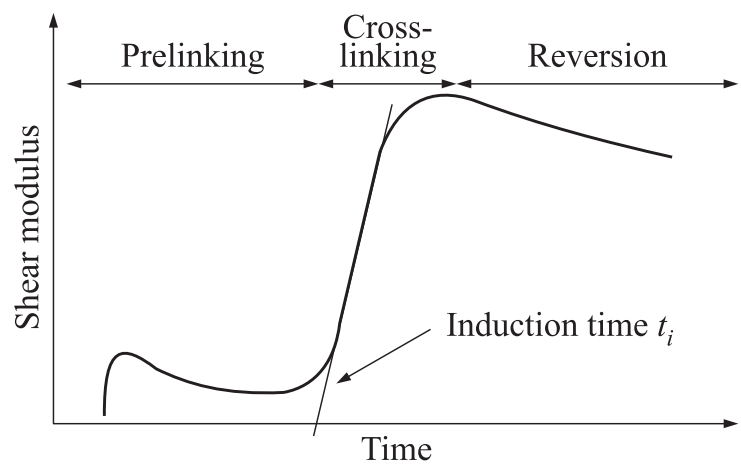

Figure 5 General shape of a rheometric curve for a sulphur-based vulcanizated elastomer 
following decrease, called reversion, is usually attributed to the destruction of polysulfidic links, the formation of inactive pendant groups, cycles, or main chain modifications.

Polysulfidic (Fig. 4a) and monosulfidic (Fig. 4b) cross-links do not exhibit a similar stability because of the different bonding energies between heterolitic bonding $\mathrm{C}-\mathrm{S}$ and homolitic bonding $\mathrm{S}-\mathrm{S}$.

Structures like $(c)$ and $(d)$ in Fig. 4 are not active for elasticity. Thus, it is important to determine the fate of sulphur atoms. The knowledge of elastomer microstructure should be a key point in understanding its macroscopic mechanical properties. Nevertheless, correlations between both scales remain difficult. looseness $=1$

\subsection{Chemical Modeling of Sulphur Vulcanization}

Classical polynomial or purely phenomenological models such as Isayev model [3] widely used for reticulation modeling fail to address correctly the main phenomena of sulphur vulcanization, i.e., induction time, acceleration of cross-linking, and reversion. The reason is that such models do not rely on any chemical consideration. One can avoid describing the prelinking chemistry, considering that vulcanization only starts once rubber has stored enough energy. This may be written in the following way:

$$
\bar{t}=\int_{0}^{t} \frac{d t}{t_{i}}
$$

where $\bar{t}$ is a dimensionless time and $t_{i}$ the induction time at a given temperature, which may be related to any convenient law such as Arrhenius law. One considers that cross-linking starts when $\bar{t}>1$.

This approach has nevertheless an important drawback: it does not take into account the fact that prelinking chemistry may affect cross-linking chemistry and the concentration of cross-links itself. Coran suggested an analytical model based on a self-accelerated mechanism to describe the first steps of prelinking chemistry [4]. This model diverges as the accelerator disappears, i.e., as cross-linking starts. A complementary analytical model developed by Ding and Leonov focuses on the cross-linking chemistry and reversion [5]. It does not seem that an univoque analytical model to describe all steps of vulcanization may be obtained. The model involves six chemical steps and six corresponding kinetic constants and can be numerically integrated (Figs. 6 and 7). The starting point of the mechanisms is the complex involving a form of dibenzothiazole polysulfide that may react with isoprene rubber. A chain with a benzothiazole polysulfide as pendant group and mercaptobenzothiazole as by-product are formed. The pendant group is dissociated to an active derivate (an alkenyl persulfenyl radical). 

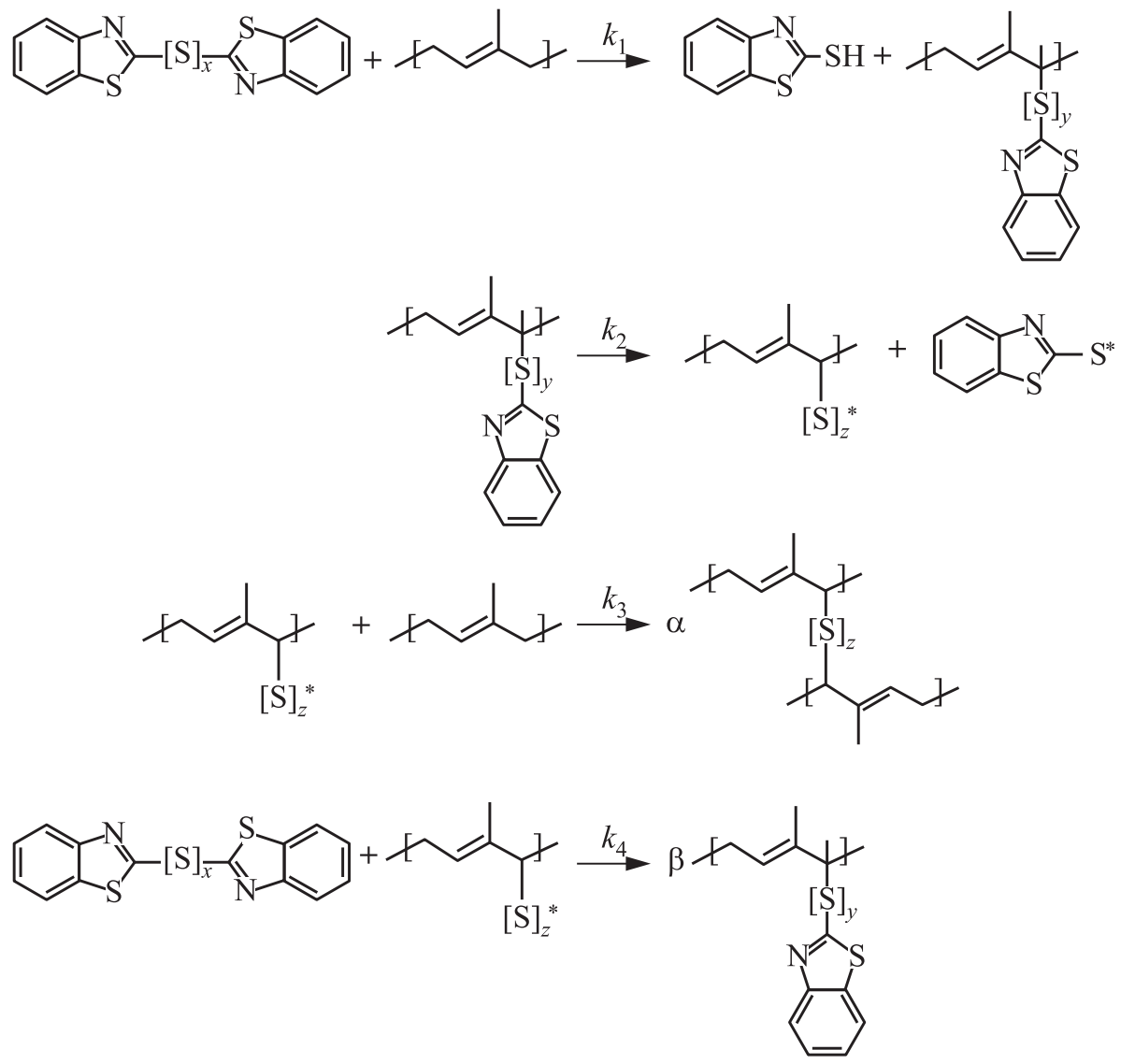

Figure 6 Main reaction pathways for sulfenamide-sulphur prelinking chemistry

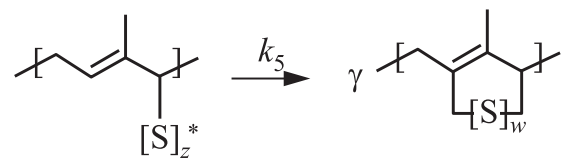<smiles>CCC=C(C)C(C)[SiH2]C(C)C(C)=CCCC</smiles>

Figure 7 Main reaction pathways for sulfenamide-sulphur reversion 
This active intermediate reacts with isoprene rubber chains to form effective cross-linking (reaction 3). Nevertheless, this last reaction is assumed to be in competition with reaction 4 in which the active intermediate reacts on the sulphuration complex to form new pendant groups on isoprene rubber chains. This reaction is supposed to be faster than reaction 3 , so that no effective cross-linking may occur until all sulphuration complex has disappeared. These competitive channels for the active intermediate may be seen as chain-branching reaction for which branching ratio is dictated by the accelerator concentration. This model can explain the long induction time and the accelerator effects observed for rubber sulphur vulcanization with sulfenamide systems.

Ding and Leonov contributed to the Coran's mechanisms by adding two other reactions: one takes into account the possibility of noneffective cross-linking when the reactive intermediate reacts on itself (reaction 5). This may justify the fact that the cure temperature affects the level of maximal shear-modulus. The last reaction represents the reversion effects through the desulphuration that affects the polysulfidic cross-links.

\subsection{Identification of the Chemical Model}

The global Coran, Ding, and Leonov model was identified for the chemical system used for rubber of the P80 flexible bearing. The numerical stiffness of the system does not allow a direct identification of the rate constants as a function of temperature. So, rheometric curves were first recorded at different temperatures. For each one, identification of the six parameters of the system was carried out. The combination of the 5th-order Runge-Kutta meth-

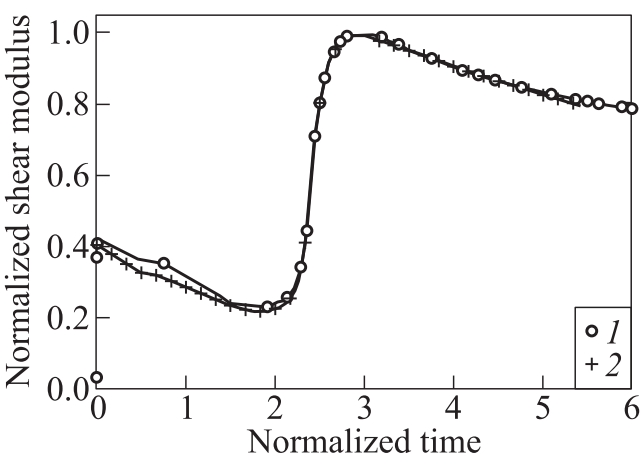

Figure 8 Validation of the chemical model on nonisothermal rheometric cycle: 1 - experiment and 2 - simulation od [1] with adaptive time step control for the numerical integration and a Levenberg-Marquardt method [1] was used to achieve a least square criterion. Each rate constant was then fitted against temperature according to the Arrhenius law. The model finally involved twelve parameters. The model was validated on a nonisothermal cure simulation cycle (Fig. 8). The agreement between predicted and measured time histories of the shear stress of the rubber is excellent. 


\section{APPLICATION TO P80 SCALE}

\subsection{Thermal Modeling of Flex-Seal Molding}

The mechanical behavior of $\mathrm{P} 80$ flex-seal bearing is mainly linked to the intrinsic mechanical properties of the rubber. The theory of elasticity indicates that mechanical properties such as shear modulus or Young modulus can be correlated to cross-link density that is very sensitive to thermal history, especially in the case of sulphur-based vulcanization process because of the strong nonlinearity and nonmonotonicity of chemical pathways. The resultant torque of the flexseal bearing requires a good control and knowledge of thermal histories and kinetics laws. The insulating character of both components of low-torque flexseal bearing (i.e., glass-epoxy shims and rubber pads) combined with its size implies necessarily strong thermal gradients in the flex-seal bearing during curing process. In such conditions, it is very difficult to guarantee a homogeneous and minimal cross-linking density in the entire flex-seal bearing to obtain the required low-torque modulus. So, a numerical simplified screening thermal analysis was performed at the P80 scale. In this analysis, only the flex-seal bearing was taken into account, the mold itself being modeled by a steel brick around the flex-seal bearing. The tested thermal cycles were treated as a time-dependent Dirichlet boundary condition applied on the mold. For each cycle, the thermal fields in the rubber pads were evaluated and postprocessed by numerical integration of the partial differential equations system describing the vulcanization process. As an output, one obtains vulcanization state and dispersion at each point of the rubber pad. Those calculations are not computer-time consuming, so it is easy to define the most suitable thermal cycle. Figure 9 depicts a typical result for the

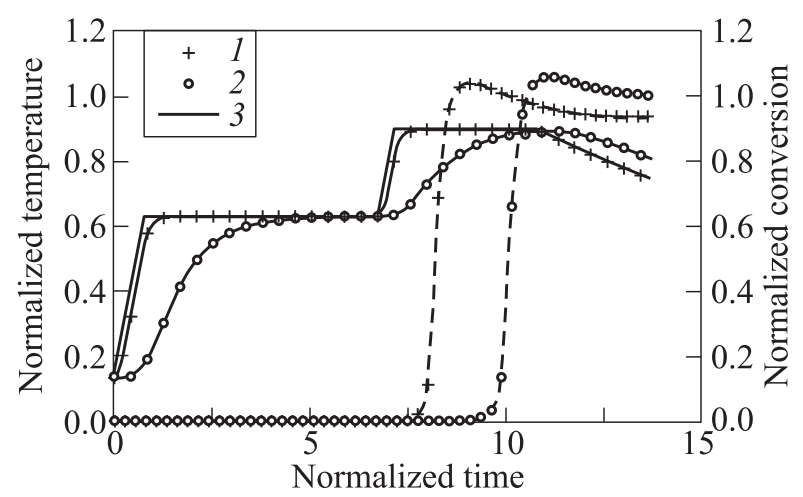

Figure 9 Thermal histories (solid curves) and vulcanization conversion rate (dashed curves) estimated in P80 flex-seal bearing: 1 - high-temperature and 2 - lowtemperature; 3 refers to Dirichlet condition 
thermal cycle used in terms of extreme thermal histories in the rubber pads. The postprocessing of those histories clearly shows that for the hottest zone in the rubber pads, the elastomer has already started its reversion, when cross-linking did not start yet in the coldest ones. The cooling step was taken into account, because it plays an important role during the process. The thermal inertia of the flex-seal bearing is used to achieve the required curing level in the coldest zones of elastomer.

The chosen thermal cycle was then experimentally tested on rubber specimens: test pieces were submitted to calculated extreme thermal histories. It was verified on those specimens that the mechanical properties were those searched. This step allowed the full-scale tests on the P80 flex-seal bearing mold. The main objective was to experimentally reproduce in the mold

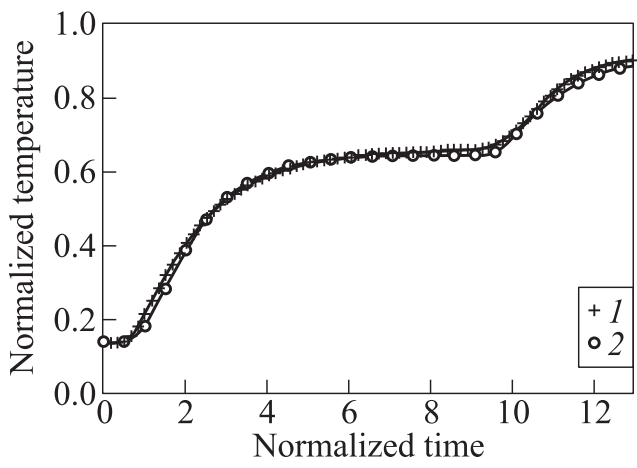

Figure 10 Lowest temperature calculated (1) and measured (2) in the rubber pads the numerically chosen Dirichlet conditions. The mold and the rubber pads were extensively equipped with thermocouples. The thermal model developed and validated for the small-scale mold was applied to the P80 flex-seal bearing mold. Figure 10 depicts, for example, the confrontation between calculated and measured thermal histories for the coldest point in the rubber pad. The agreement is very good.

\section{CONCLUDING REMARKS}

The development of P80's flex-seal bearing manufacturing process relies on a new methodology to avoid time and cost-consuming experimental iterative steps. Numerical modeling plays a major role in the definition of the curing process of flex-seal bearing. A model was set up for the description of the thermal comportment of a complex resistive-heated mold. The model was validated on a small-scale mold and provided the temperature histories of the elastomer during the complete process.

A chemical model was also setup to accurately describe the cross-linking chemistry of a sulphur and sulfenamide system. With this model, it is possible to predict the shear modulus of the elastomer as a function of thermal history. The model was identified and validated for the P80's flex-seal bearing newly developped elastomer. 
Both thermal and chemical aspects combined together were applied at the P80 scale. The final model was very helpful in designing thermal process cycles to obtain the required low-torque module and to avoid difficulties and dispersions due the scale and insulating character of P80 flex-seal bearing.

Should any nonconformances related to abnormal manufacturing cycle appear during serial production, this modeling tool is expected to facilitate decision making with respect to reliability, delay, and cost aspects.

\section{ACKNOWLEDGMENTS}

This study was made possible with external helps. The authors strongly acknowledge:

- ESA/CNES Research \& Technologies department for its support in the frame of the P80 nozzle program development;

- DGA (General Delegation for Armament) for its strong and steady support in material development; and

- TECHLAM for its manufacturing expertise.

\section{REFERENCES}

1. Press, W. H., S. A. Teukolsky, W. T. Vetterling, and B. P. Flannery. 1992. Numerical recipes in F77. 2nd ed. Cambridge University Press.

2. Taine, J., and J.P. Petit. 1989. Transferts thermiques: Mécanique des fluides anisothermes. Dunod: Dunod Université.

3. Chan, T. W., G. D. Shyu, and A. I. Isayev. 1995. Master curve approach to polymer crystallization kinetics. Polym. Eng. Sci. 35:733-40.

4. Coran, A. 1964. Vulcanization. Part VI. A model and treatment for scorch delay kinetics. Rubber Chem. Technol. 37(3):689-97.

5. Ding, R., and A. I. Leonov. 1996. A kinetic model for sulphur accelerated vulcanization of a natural rubber compound. J. Appl. Polym. Sci. 61(3):455-63. 